\title{
Social selection in seeking medical care for reduced fecundity among women in Denmark
}

\author{
PAMELA RACHOOTIN AND JØRN OLSEN \\ From the Department of Social Medicine, Institute of Community Health, University of Odense, Denmark
}

SUMMARY This study is based on a representative sample of 709 Danish women aged between 25 and 45 who were interviewed in 1979. The purpose was to estimate the proportion of women with reduced fecundity who seek medical care and to identify sociodemographic variables associated with presentation to the medical care system. The study showed that the majority of women with reduced fecundity did not seek medical care. The propensity to seek care was not significantly associated with a woman's age or education, nor with family income or the employment status of the head of the household. Women living in rural areas or in homes with two or more rooms per family member had a greater tendency to seek medical care for secondary reduced fecundity compared with women living in cities or in more crowded homes. The implications of these findings for epidemiological studies of the association of reduced fecundity and occupation are discussed.

Epidemiologic studies on reduced fecundity are surprisingly sparse. An earlier review of these studies $^{1}$ suggested that certain environmental agents act to reduce fecundity and that exposure to such agents often takes place at work. Since only a small proportion of the thousands of possible agents harmful to fecundity have been investigated, a great deal of further work in this area is necessary.

Retrospective case-control studies can play an important role in identifying hazards. A necessary prerequisite to conducting such studies is an adequate understanding of the selection mechanisms which operate to define study groups. The two specific aims of the present study were to estimate the proportion of women with reduced fecundity who seek medical care, and to identify sociodemographic variables that correlate with care-seeking behaviour so as to define potential selection bias.

\section{Methods}

The data were collected as part of a national survey administered jointly by the Danish National Bureau of Statistics and the Danish National Institute of Social Research in October, 1979. A two-stage stratified random sampling procedure was followed to obtain a sample representative of the Danish population in terms of geographic distribution, degree of urbanisation, and occupational structure. ${ }^{2}$ In the first stage, municipalities were selected on the basis of their geographic location, degree of urbanisation, and occupational structure. In the second stage, a sample proportional to the total number of persons aged 16 and over was chosen in each selected municipality. The enumerated sample included 1.4 persons per 1000 residents in Denmark over the age of 16 . Only women between the ages of 25 and 45 were eligible for inclusion in the present study. Of 953 women who fulfilled the age criterion of the present study, $709(74 \%)$ were interviewed, $170(18 \%)$ refused, and $74(8 \%)$ could not be contacted or interviewed for miscellaneous reasons (illness, non-Danish speaking, missed appointment, etc.). It should be noted that the refusals applied to participation in the general survey, not specifically to the subset of interview items upon which the present study is based. The response rate to the general survey was highest among women aged 25 to 29 (78\%) and lowest among women aged 40 to 45 (71\%).

The interviews included questions on total number of pregnancies, conception time and pregnancy outcome, unsuccessful efforts to conceive, and earlier medical consultations for problems in achieving conception. In addition, background information was collected, including age, marital status, family size, occupation, education, income, housing conditions, and place of residence.

Women who had failed to achieve a first pregnancy after engaging in sexual activity without using contraceptive methods for a period of two years or longer were defined as having primary reduced 
fecundity (PRF). This definition was applied regardless of whether a pregnancy was eventually achieved. The term secondary reduced fecundity (SRF) was applied to women who failed to achieve a second or subsequent pregnancy within a two-year period of cohabitation without the use of contraceptive methods.

\section{Results}

Sixty-three women fulfilled the criteria for PRF (approximately 9\% of the interviewed women) and 64 fulfilled the criteria for SRF. Thirteen women were represented in both categories.

The Figure shows that less than half of the women with PRF sought help from a general practitioner and less than a third with PRF were referred to a hospital. Among the group with SRF even fewer sought medical care.

The analysis concentrated on comparing infertile women who had sought hospital care with those who had not. This comparison was thought to be particularly important because future

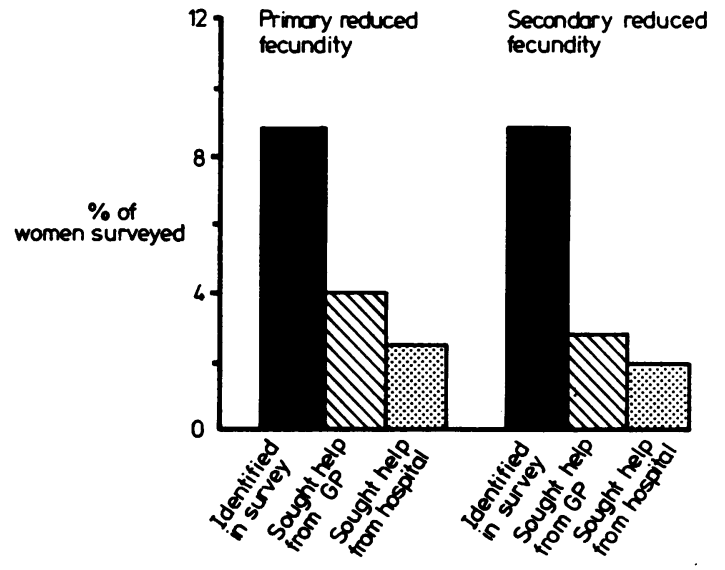

Figure Overall frequency of primary and secondary reduced fecundity and frequency of seeking medical care

epidemiological studies are likely to recruit infertile subjects identified from hospital sources. Table 1 presents the results of a logistic regression ${ }^{3}$ to examine whether certain demographic and

Table 1 Logit analysis of hospital care sought for reduced fecundity

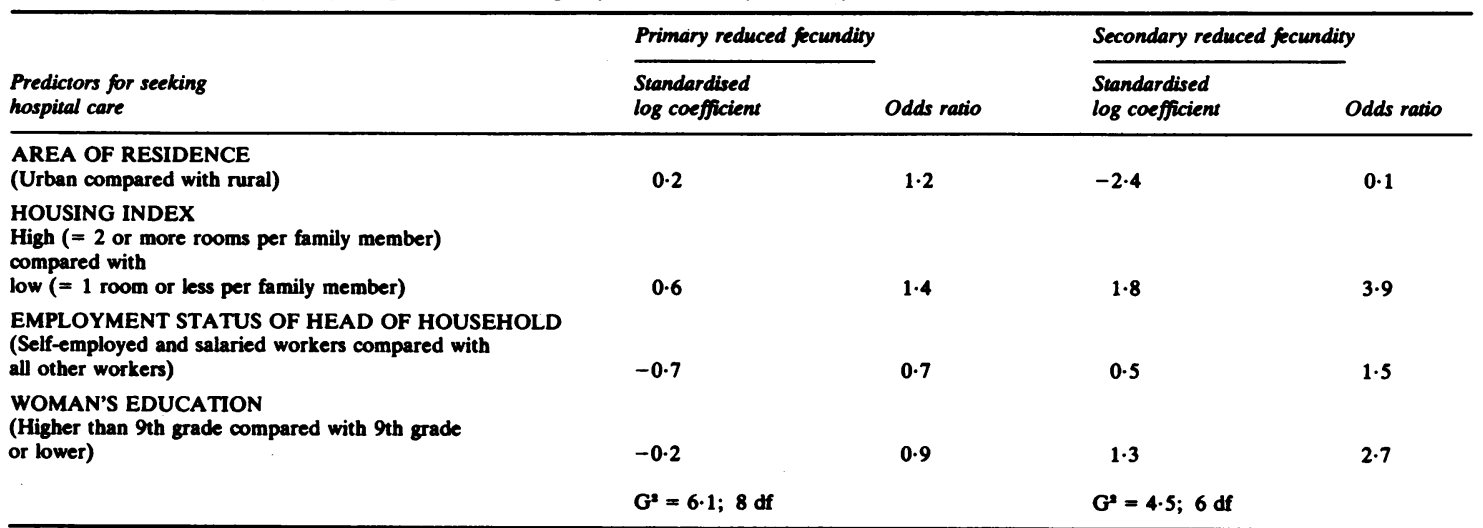

The standard group for comparison is defined as having a rural residence, low housing index, low employment status, and low level of educational attainment. In this group the odds of hospital care-seeking compared with non-hospital care-seeking is 0.5 for women with primary reduced fecundity and 0.3 for women with secondary reduced fecundity.

Table 2 Sociodemographic characteristics of women with a history of reduced fecundity by hospital care-seeking behaviour

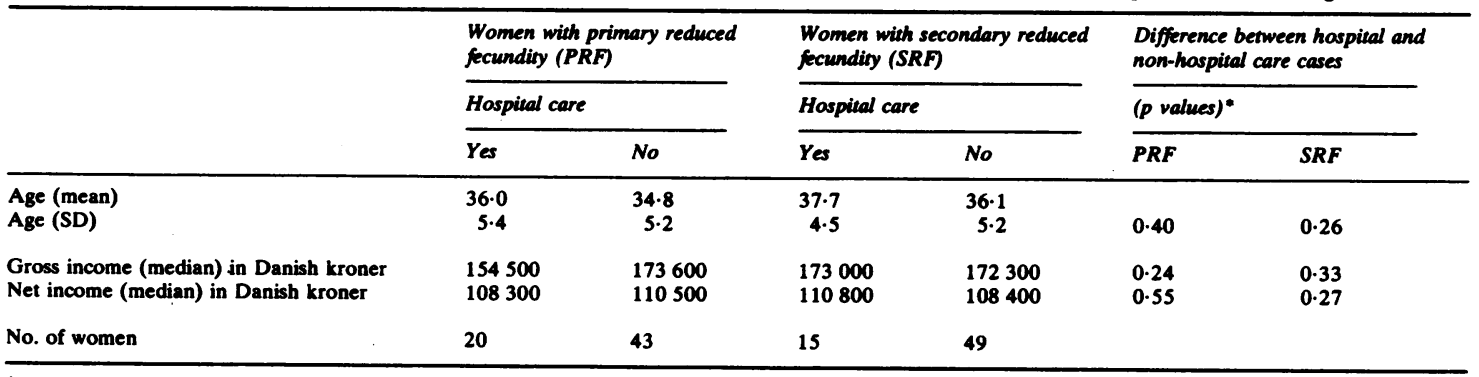

* Mann-Whitney U-test. 
socioeconomic variables are associated with hospital care-seeking among women with reduced fecundity. None of the variables examined were meaningful predictors of care-seeking among the group with PRF. Among the group with SRF there was a significantly greater tendency to seek hospital care among women residing in rural compared with urban areas $\left(\mathrm{G}^{2}=6.0 \mathrm{df}=1 \mathrm{p}<0.05\right)$. In addition, more women sought hospital care in the group that lived in a home with two or more rooms per family member compared with women living in relatively more crowded homes. This difference, however, was not statistically significant at the $5 \%$ level $\left(\mathrm{G}^{2}=3.6\right.$ $\mathrm{df}=1 \quad 0.05<\mathrm{p}<0 \cdot 10)$. Women's education and head-of-household's employment status played a lesser $(p>0 \cdot 10)$ role in hospital care-seeking for SRF.

Table 2 shows that the average age at interview was not significantly higher among the group who had sought hospital care than among those who had not. Family income was almost identical in the hospital care-seeking and non-hospital-care-seeking groups. (It must be noted that information on income was unknown for $34 \%$ of the interviewed women. In the analysis missing values were uniformly replaced with the median values found for the women who were able to provide information on family income).

\section{Discussion}

The findings of this study seem to justify caution in the recruitment of subfecund women from hospital settings in Denmark. The minority of women who do seek hospital care for SRF are not completely representative of all women with this medical problem, at least in terms of the variables which were studied. There is a definite bias towards rural women among those seeking hospital care for SRF. This is surprising in view of the fact that women in rural areas have to travel greater distances to hospitals than urban residents. There is also a bias towards those living in less crowded housing conditions. These factors may be less important for PRF than for SRF. The precise relationship of socioeconomic status to care-seeking behaviour is difficult to determine from the present study, however, because income, education, and employment status of the 'head of the household' were not strongly related to seeking care at a hospital. It is likely that further studies using larger sample sizes could further clarify the issue of social variables and care-seeking behaviour of subfecund women.

The present study is the first in a series of studies of occupational exposures associated with reduced fecundity. Because such exposures are likely to be distributed differently in different social groups and between residents in urban and rural areas, it was important to evaluate whether sociodemographic factors also influence the likelihood of a diagnosis of infertility (in this case by presentation for medical care). Generally, when a factor exists which is associated both with exposure and the likelihood of diagnosis there is a potential for confounding or bias in a study.

Our results do not explain why some subfecund women seek medical care while others do not. This was not the objective of the study. It will be interesting in future studies to ask subfecund women directly why medical care was or was not sought. In this study the only factor found which might explain care-seeking behaviour was the number of live births experienced before an episode of SRF. Specifically, the greater the number of previous live births, the less likely women were to seek medical care for an episode of SRF $\left(\chi^{2}\right.$ test for trend $=7.041 \mathrm{df}$ $\mathrm{p}<0.01$ ). There may also be a number of complex psychological factors which influence care-seeking behaviour. It is doubtful, however, whether such factors would be related to a woman's likelihood of exposure. In any event, the current lack of knowledge of the reasons behind self-selection for hospital care does not in itself prevent scientifically acceptable case-control studies of the occupational aetiology of infertility from being conducted in the future.

Reprints from Ms. Pamela Rachootin, Department of Social Medicine, Institute of Community Health, University of Odense, J.B. Winsløws Vej 17, 5000 Odense C, Denmark.

\section{References \\ ${ }^{1}$ Rachootin P. Eksogene årsager til infertilitet. En litteraturgennemgang. Ugeskr Lag 1980; 142: 1537-40. \\ ${ }^{2}$ Socialforskningsinstituttet og Danmarks Statistik. Orientering om Omnibusundersøgelserne. Copenhagen: Stencil, 1977. \\ ${ }^{3}$ Bishop YMM, Feinberg SE, Holland PW. Discrete multivariate analysis: Theory and practice. Cambridge, Mass: MIT Press, 1975.}

